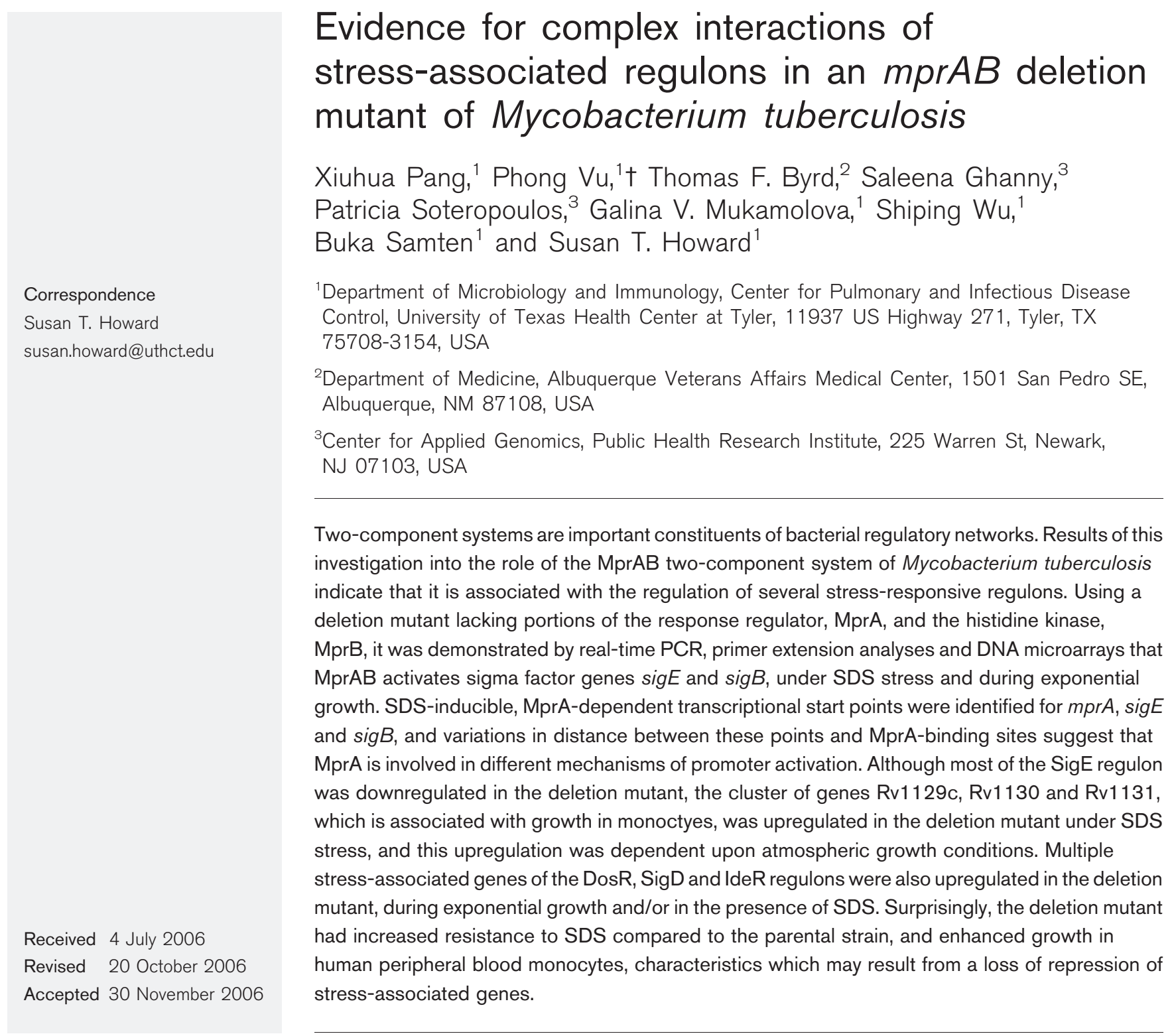

\title{
Evidence for complex interactions of stress-associated regulons in an mprAB deletion mutant of Mycobacterium tuberculosis
}

\author{
Xiuhua Pang, ${ }^{1}$ Phong Vu, ${ }^{1} \dagger$ Thomas F. Byrd, ${ }^{2}$ Saleena Ghanny, ${ }^{3}$ \\ Patricia Soteropoulos, ${ }^{3}$ Galina V. Mukamolova, ${ }^{1}$ Shiping $\mathrm{Wu},{ }^{1}$ \\ Samten 1 and Susan T. Howard \\ 75708-3154, USA \\ 2Department of Medicine, Albuquerque Veterans Affairs Medical Center, 1501 San Pedro SE \\ c7108, USA \\ ${ }^{3}$ Center for Applied Genomics, Public Health Research Institute, 225 Warren St, Newark \\ NJ 07103, USA
}

\begin{abstract}
Two-component systems are important constituents of bacterial regulatory networks. Results of this investigation into the role of the MprAB two-component system of Mycobacterium tuberculosis indicate that it is associated with the regulation of several stress-responsive regulons. Using a deletion mutant lacking portions of the response regulator, MprA, and the histidine kinase, $\mathrm{MprB}$, it was demonstrated by real-time PCR, primer extension analyses and DNA microarrays that MprAB activates sigma factor genes $\operatorname{sig} E$ and $\operatorname{sig} B$, under SDS stress and during exponential growth. SDS-inducible, MprA-dependent transcriptional start points were identified for $m p r A$, sigE and $\operatorname{sig} B$, and variations in distance between these points and MprA-binding sites suggest that MprA is involved in different mechanisms of promoter activation. Although most of the SigE regulon was downregulated in the deletion mutant, the cluster of genes Rv1129c, Rv1130 and Rv1131, which is associated with growth in monoctyes, was upregulated in the deletion mutant under SDS stress, and this upregulation was dependent upon atmospheric growth conditions. Multiple stress-associated genes of the DosR, SigD and IdeR regulons were also upregulated in the deletion mutant, during exponential growth and/or in the presence of SDS. Surprisingly, the deletion mutant had increased resistance to SDS compared to the parental strain, and enhanced growth in stress-associated genes.
\end{abstract}

\section{INTRODUCTION}

As evidenced by its historical and current impact on human populations (Corbett \& Raviglione, 2005; Zink et al., 2005), Mycobacterium tuberculosis is a highly successful pathogen. To withstand the challenges of aerosol transmission, growth within host macrophages, and prolonged encapsulation within lung granuloma, these organisms require the ability

tPresent address: UT Southwestern Medical Center, Dallas, TX, USA. Abbreviations: EMSA, electrophoretic mobility shift assay; iVEGI, in vivo-expressed genomic island; TCS, two-component system; TSP, transcriptional start point.

The GEO accession numbers for the array data associated with this paper are GSM155424-155447 (series record no. GSE6750).

Four supplementary tables are available with the online version of this paper. to respond to different types of stress. M. tuberculosis has 13 sigma factors (Cole et al., 1998), and DNA microarray analyses have shown the importance of several of these in regulating the changes in gene expression patterns associated with various stresses (Geiman et al., 2004; Manganelli et al., 2001, 2002). In addition to sigma factors, the response regulators of some two-component systems (TCSs) (Rison et al., 2005), such as DosR (Kendall et al., 2004; Park et al., 2003) and PhoP (O’Toole et al., 2003; Perez et al., 2001; Walters et al., 2006), and other transcriptional regulators, such as IdeR (Dussurget et al., 1999; Manabe et al., 2005) and EmbR (Sharma et al., 2006), have been shown to modulate mycobacterial gene expression in response to particular stresses. However, in general, the mechanisms by which $M$. tuberculosis senses a particular stress, and activates the appropriate regulatory factor(s) while inhibiting others, are largely unknown. 
We are investigating the role of the MprAB TCS of $M$. tuberculosis in the stress response. TCSs are histidine phosphotransfer systems involved in sensing environmental changes and triggering adaptive responses (Hoch \& Varughese, 2001). Studies indicate that MprA is an autoregulatory transcription factor, which is phosphorylated by the histidine kinase MprB (He \& Zahrt, 2005; Zahrt et al., 2003). Results of genome-wide transposon mutagenesis suggested that $m p r B$ was essential for growth in broth, whereas $m p r A$ was nonessential (Sassetti et al., 2003).

$m p r A$ and $m p r B$ are part of the iVEGI (in vivo-expressed genomic island) region, which is highly upregulated during growth of M. tuberculosis in mice (Talaat et al., 2004). Many iVEGI genes are predicted to be involved in cell wall biosynthesis and lipid metabolism (Talaat et al., 2004), and a recent study suggests that they are important in $M$. tuberculosis infection of the central nervous system (Jain et al., 2006). The role of MprA in survival is unclear, however, as an mprA insertion mutant had reduced persistence in a murine model, but increased growth in resting murine macrophages (Zahrt \& Deretic, 2001).

$m p r A$ and $m p r B$ are also upregulated by SigE, during exposure to the detergent SDS (Manganelli et al., 2001). SigE is a major stress-responsive sigma factor of $M$. tuberculosis (Jensen-Cain \& Quinn, 2001; Manganelli et al., 1999, 2001; Raman et al., 2001), and often functions within a regulatory cascade (Manganelli et al., 2002; Raman et al., 2001). SDS exposure upregulates SigE, which then activates 23 genes, including sigB, $m p r A, m p r B$ and four other transcription factor genes (Manganelli et al., 2001). Some SDS-activated genes are also upregulated during growth of $M$. tuberculosis in macrophages, suggesting that, like SDS exposure, macrophages may damage lipid components of the bacterial envelope (Schnappinger et al., 2003). Similar to rpoE of Escherichia coli (Mecsas et al., 1993; Tam \& Missiakas, 2005), M. tuberculosis sigE may, therefore, be induced by envelope stress, but the mechanisms involved have not been determined. Deletion of sigE in mycobacteria is associated with reduced resistance to SDS and oxidative stress, decreased growth in macrophages (Manganelli et al., 2001; Wu et al., 1997), and reduced growth in mice (Ando et al., 2003; Manganelli et al., 2004).

As MprAB is the only TCS upregulated by SigE under SDS stress, we hypothesized that MprAB may be involved in sensing cell envelope damage and upregulating genes within the SigE regulon. In this study, using a deletion mutant in which both $m p r A$ and $m p r B$ were disrupted, we determined that MprA regulates both sigE and $\operatorname{sig} B$, during normal growth conditions and under SDS stress. We also identified SDS-inducible transcriptional start points for $m p r A$, sigE and $\operatorname{sig} B$. In addition, similar to a recent report using an mprA insertion mutant (He et al., 2006), we observed that, in the MprAB deletion mutant, much of the SigE regulon was downregulated under SDS stress. However, further analyses indicated that a cluster of genes within the SigE regulon is highly upregulated in the absence of MprAB under SDS stress, and that this upregulation is sensitive to atmospheric growth conditions. Moreover, multiple genes from regulons associated with hypoxia, starvation and iron metabolism are upregulated in the MprAB deletion mutant. Unexpectedly, the deletion mutant had enhanced survival in SDS, as well as increased growth in human peripheral blood monocytes. These data suggest that the MprAB TCS is an important link within a complex network of stressassociated regulons involved in survival of M. tuberculosis.

\section{METHODS}

Bacterial strains and culture conditions. M. tuberculosis mutants and parental strain $\mathrm{H} 37 \mathrm{Rv}$ were grown in either Middlebrook $7 \mathrm{H} 9$ broth containing $0.05 \%$ Tween 80 , or Middlebrook 7H10 agar (Difco), both enriched with $10 \%$ oleic acid-albumindextrose-catalase (OADC; Difco). Broth cultures were incubated at $37^{\circ} \mathrm{C}$ with gentle shaking, under normal atmospheric conditions (without additional $\mathrm{CO}_{2}$ ), except where indicated. E. coli Novablue and Rosetta(DE3)pLysS (Novagen) were used as host strains for general cloning and gene expression, respectively. E. coli strains were grown on $\mathrm{L}$ agar or in $\mathrm{L}$ broth. Antibiotics were added to growth media as required.

Construction of the mprAB deletion mutant, Rv-D981. Sequences for primers used in this study are included in Supplementary Table S1, available with the online version of this paper. An 1150 bp deletion, which included the C-terminal half of MprA and the N-terminal half of MprB, was generated in H37Rv using counterselectable markers (Parish \& Stoker, 2000), and plasmids kindly provided by T. Parish (Queen Mary's School of Medicine and Dentistry, London, UK). Briefly, two genomic fragments of approximately $1 \mathrm{~kb}$ were amplified, inserted into pGEM-TEasy (Promega), and sequenced. Fragment A, generated using primers D981S-5 and D981S-3, begins within Rv0980c and ends $300 \mathrm{bp}$ downstream of the predicted start codon of mprA. Fragment B, generated using primers D982E-5 and D982E-3, begins $800 \mathrm{bp}$ downstream of the $m p r B$ start codon and ends within Rv0983 $(p e p D)$. Fragment B was inserted into p2NIL using a ScaI/KpnI digest, yielding plasmid pN982E, and then fragment A was inserted into pN982E, using a PstI/ScaI digest, producing pD981. The selection cassette from pGOAL17 was inserted into the PacI site of pD981, producing $\mathrm{pD} 981 \mathrm{P}$, which was used to generate the deletion mutant Rv-D981 from H37Rv, as described by Parish \& Stoker (2000). The deletion in Rv-D981 was verified by Southern blotting and PCR.

Complementation of Rv-D981. A $2.8 \mathrm{~kb}$ genomic region containing $m p r A, m p r B$ and the intergenic region between $m p r A$ and Rv0980c was amplified using primers 981UP5A and 982XP-3. The amplicon was inserted into pSTBlue-1 (Novagen), sequenced, and then the insert was released by digestion with SnaBI, which cuts in the vector, and XbaI. The gel-purified insert was ligated into the integrative vector pMV361 (Stover et al., 1991), which was precut with $\mathrm{HpaI}$ and $\mathrm{XbaI}$. The resulting plasmid, p981-COM, was electroporated into strain Rv-D981, followed by plating and selection for kanamycin-resistant colonies. The presence of the intact MprAB region, in the complemented strain Rv-D981C, was confirmed by PCR.

Construction of an mprA expression plasmid and purification of MprA. The $690 \mathrm{bp}$ predicted coding region of mprA was amplified by PCR using primers MprA-P5 and MprA-P3, and inserted into pSTBlue-1, generating pSTH18. Following sequence verification, a BamHI/HindIII fragment of pSTH18 was ligated into BamHI/HindIII-cut pBEn-SBP-SETla (Stratagene), a Variflex 
expression vector containing an N-terminal streptavidin-binding peptide tag and a solubility enhancement tag. The resulting plasmid, pSTH20, was used to transform E. coli Rosetta(DE3)pLysS (Novagen). Expression of mprA was induced by the addition of IPTG with incubation for $3 \mathrm{~h}$ at $30^{\circ} \mathrm{C}$. Bacteria were collected by centrifugation at $4{ }^{\circ} \mathrm{C}$, and then resuspended in $1 \mathrm{ml}$ streptavidinbinding buffer $(10 \mathrm{mM}$ Tris/HCl, $\mathrm{pH} 8.0,150 \mathrm{mM} \mathrm{NaCl})$. Following sonication, lysates were centrifuged for $5 \mathrm{~min}$ at 13000 r.p.m. and the tagged MprA was recovered from the supernatant using streptavidin agarose (Novagen), according to the manufacturer's instructions. Peptide tags were removed using the Thrombin CleanCleave kit (Sigma), and removal was verified by gel electrophoresis of the MprA protein before and after cleavage.

Electrophoresis mobility shift assays (EMSAs). The ability of MprA to bind DNA was confirmed using the mprA promoter (data not shown). To analyse binding of MprA to the sigE promoter, probes P1, P2 and P3 were amplified using primer pairs SigEGST-1/ SigEGST-2, SigEGST-1/SigEGST-6 and SigEGST-2/SigEGST-5, respectively. SigE45-F/SigE45-R, SigE30-F/SigE30-R and SigE54-F/ SigE54-R were annealed to generate probes P4, P5 and P6, respectively (see Table $\mathrm{S} 1$ for primer sequences). Progressively shorter sections were generated using the following oligonucleotide pairs: $\operatorname{SigE}+2 \mathrm{~F} / \mathrm{SigE}+2 \mathrm{R}$ (probe $\mathrm{P} 7), \operatorname{SigE}+5 \mathrm{~F} / \mathrm{SigE}+5 \mathrm{R}$ (probe P8), and $\mathrm{SigE}+8 \mathrm{~F} / \mathrm{SigE}+8 \mathrm{R}$ (probe P9) (see Results for details). sigB promoter probes were amplified using the following primer pairs: SigBGST-4/SigBGST-3 (probe P1), SigBGST-4/SigBGST-2 (probe P2), SigBGST-1/SigBGST-3 (probe P3), and SigBGST-1/SigBGST-2 (probe P4). Primers SigBGST-5 F/R and SigBGST-6 F/R were annealed to generate $\operatorname{sig} B$ probes $\mathrm{P} 5$ and $\mathrm{P} 6$, respectively. DNA probes were end-labelled with $\left[\gamma_{-}{ }^{32} \mathrm{P}\right] \mathrm{ATP}$ using T4 polynucleotide kinase (Promega) and were separated from free isotope using Quick Spin Column (Roche) filtration. Labelled DNA probes were incubated with MprA using described procedures (Samten et al., 2002). For competition assays, 100-, 200- or 400-fold excess (in ng) of unlabelled competitor DNA was included. Reaction mixtures were loaded onto 5-6\% nondenaturing polyacrylamide gel and electrophoresed for $2-3 \mathrm{~h}$ at $140 \mathrm{~V}$ at $4{ }^{\circ} \mathrm{C}$ in $0.5 \times$ TBE buffer. A $278 \mathrm{bp}$ fragment, which was located near Rv0980c, did not bind MprA and was used as a negative control in competition assays.

RNA isolation. Mycobacterial strains were cultured in $7 \mathrm{H} 9$ medium to mid-exponential phase $\left(\mathrm{OD}_{600} 0.4-0.5\right)$ at $37^{\circ} \mathrm{C}$ with shaking, in the absence of $\mathrm{CO}_{2}$, except as indicated. Total RNA was isolated using TRIzol LS Reagent (Invitrogen) according to the manufacturer's instruction, except that lysing matrix B and a FastPrep FP120 shaker (BIO 101) were used to disrupt the mycobacteria. With large culture volumes, bacteria were first pelleted by centrifugation and resuspended in a small volume of $0.2 \%$ Tween, prior to adding to the lysing tubes. Chromosomal DNA was removed with 'DNA-free' reagents (Ambion), according to the manufacturer's instructions. For experiments with detergent stress, cultures were grown to exponential phase $\left(\mathrm{OD}_{600} 0.3-0.4\right)$, SDS was added to a final concentration of $0.05 \%$, and RNA was extracted from control and SDS-treated samples after $90 \mathrm{~min}$ incubation at $37^{\circ} \mathrm{C}$.

Primer extension analysis. Primers 981PE1 and 981PE3 were used to analyse mprA transcripts, and SigEPE1 and SigEPE2 were used to analyse TSP4 and TSP3, respectively, of the sigE promoter. Primers were labelled with $\left[\gamma-{ }^{32} \mathrm{P}\right]$ ATP by T4 polynucleotide kinase as indicated above. For the annealing step, $17 \mu \mathrm{g}$ of $M$. tuberculosis RNA was denatured at $90{ }^{\circ} \mathrm{C}$ for $5 \mathrm{~min}$, snap-cooled on ice, and then incubated for $20 \mathrm{~min}$ with 2 pmol of labelled primers in $1 \times$ reverse transcription buffer $[50 \mathrm{mM}$ Tris/HCI ( $\mathrm{pH} 8.3), 50 \mathrm{mM} \mathrm{KCl}$, $10 \mathrm{mM} \mathrm{MgCl}_{2}, 10 \mathrm{mM}$ DTT, $1 \mathrm{mM}$ each dNTP and $0.5 \mathrm{mM}$ spermidine]. Annealing temperatures were adjusted for each primer. Primer extension was performed using the Primer extension system-AMV reverse transcriptase kit (Promega) as directed by the manufacturer, and reaction products were separated using an $8 \%$ polyacrylamide sequencing gel. For sigE and $m p r A$ analysis, sequencing reactions, performed with the same primer as used in primer extension, were run in adjacent lanes to determine the start site of the transcripts.

Reverse transcription and relative quantification of mRNA by real-time PCR. Total RNA $(3 \mu \mathrm{g})$ was annealed with $2 \mu \mathrm{g}$ of random hexamer primers (pdN6, Amersham Pharmacia Biotech) and 20 units of Rnasin RNase inhibitor (Promega). Following incubation at $65^{\circ} \mathrm{C}$ for $5 \mathrm{~min}$, reverse transcription was carried out at $42{ }^{\circ} \mathrm{C}$ for $60 \mathrm{~min}$, using $200 \mathrm{U}$ M-MLV reverse transcriptase (Invitrogen), $20 \mu \mathrm{M}$ dNTPs (Roche) and first-strand buffer (Invitrogen).

Primers and probes for real-time PCR were designed with PRIMER EXPRESS software (Applied Biosystems), and probes were labelled with 5'-fluorescein phosphoramidite and 3'TAMRA. Assays were performed using an ABI Prism 7700 thermal cycler with $25 \mu$ reaction volumes containing $1 \times$ Taqman universal PCR master mix (ABI), $0.3 \mathrm{mM}$ each primer, $0.2 \mathrm{mM}$ probe and $2.5 \mu \mathrm{lDNA}$ or genomic DNA as template, and the following thermal cycles: 2 min at $50{ }^{\circ} \mathrm{C}$, $10 \mathrm{~min}$ at $95^{\circ} \mathrm{C}$ followed by 40 repeats of $15 \mathrm{~s}$ at $95^{\circ} \mathrm{C}$ and $1 \mathrm{~min}$ at $60^{\circ} \mathrm{C}$. Relative quantities of cDNA were determined from standard curves generated by amplification of serial 10-fold dilutions of H73Rv genomic DNA, using the appropriate probe and primers, and were normalized for amounts of $16 \mathrm{~S}$ rRNA. The following primer/probe sets were used: mprA (981QF, 981QR, probe 981QP); sigB (SigBQF, SigBQR, probe SigBQP); sigE set 1 (WSigEF, WSigER, probe WSigEP); 16S rRNA (W16SF, W16SR, probe W16SP).

In vitro stress assays. Cultures were grown to early exponential phase $\left(\mathrm{OD}_{600}<0.2\right)$, and divided into two $25 \mathrm{ml}$ portions. SDS was added to one portion for each strain to a final concentration of $0.05 \%$. Samples were incubated at $37^{\circ} \mathrm{C}$, and then aliquots were removed at $2 \mathrm{~h}$ or $5 \mathrm{~h}$, centrifuged to remove SDS, and then diluted in PBS prior to plating in duplicate. Colonies were counted after 2 weeks to determine percentage survival in the stress-treated samples compared to untreated controls. (Incubation for an additional 1-2 weeks did not produce any changes in colony numbers.) Resistance to SDS was examined in three separate experiments.

Infection of human monocytes. Human mononuclear cells were isolated from buffy coats purchased from United Blood Services and cultured as described by Byrd \& Horwitz (1989). After 48 h, cells were harvested and monocytes isolated by adherence to Primaria flat-bottomed wells (24 multiwell Falcon plates, Becton Dickinson), in Iscoves medium containing $10 \%$ normal human serum at concentrations of approximately $1 \times 10^{5}$ monocytes per well $(500 \mu \mathrm{l})$ for $90 \mathrm{~min}$ in $5 \% \mathrm{CO}_{2} / 95 \%$ air at $37^{\circ} \mathrm{C}$. Following wash steps and readdition of medium, monocyte monolayers were maintained under these conditions for an additional $48 \mathrm{~h}$, prior to further washing and infection using a low-inoculum assay as described by Byrd (1997). Unopsonized M. tuberculosis strains were added to monocyte monolayers at a concentration of $2 \times 10^{4}$ bacteria per well (bacteria/ monocyte ratio of $0.2: 1$ ), and the monolayers were incubated at $37^{\circ} \mathrm{C}$ in $5 \% \mathrm{CO}_{2} / 95 \%$ air. At 2, 4 and 7 days, culture supernates and cell lysates were plated separately on 7H11 agar, and values were added together to give total c.f.u. per well at each time point (Byrd, 1997). For Rv-D981, supernate c.f.u. were $6.4 \%, 5.6 \%$ and $11 \%$ of the total c.f.u. at 2, 4 and 7 days, respectively, indicating a relatively small contribution of supernate c.f.u. to total well c.f.u. The increase in supernate c.f.u. at 7 days was likely due to detached, infected monocytes in the supernate, as there was a concomitant decrease in adherent monocytes between days 4 and 7. The same relationship between groups was apparent when only c.f.u. in monocyte lysates were compared, and when c.f.u. in monocyte lysates were corrected for the number of monocytes remaining adherent at each time point (data not shown). Data were compared by Student's $t$ test. 
The viability of infected monocyte monolayers and the number of nuclei per well was also determined at each time point as described by Byrd (1997). Monocyte monolayers were $>98 \%$ viable in all groups at 2 and 4 days, with viability decreasing to a mean of $87 \%$, $69 \%$ and $96 \%$ in H37Rv, Rv-D981 and Rv-D981C, respectively, at 7 days. This decrease in viability corresponded to increasing c.f.u. and was likely due to intracellular multiplication resulting in death of infected monocytes.

DNA microarray analyses. The $M$. tuberculosis microarray consists of 4295 70-mer oligonucleotides representing the 3924 predicted open reading frames of the $\mathrm{H} 37 \mathrm{Rv}$ strain (http://www.sanger.ac.uk) with an additional 371 probes designed to detect sequences present in the CDC1551 strain (http://www.tigr.org). The arrays were prepared by spotting oligonucleotides (Tuberculosis Genome Set version 1.0, Operon Biotechnologies) onto poly-L-lysine-coated glass microscope slides using a GeneMachines Omnigrid 100 Arrayer (Genomic Solutions) and SMP3 pins (Telechem). Total RNA from three independent experiments was prepared as described above. Briefly, cDNA was synthesized using random primers and labelled with Cyanine-3 or Cyanine-5 dUTP (PerkinElmer) by a modification of the procedure described by Voskuil et al. (2003) and hybridized to the arrays overnight. For each pair of samples in each experiment, dye flips were performed. After washing, the arrays were scanned with a GenePix4000B scanner (Molecular Devices). The images were processed using GenePix 5.1. Data were filtered by removing all spots that were below the background noise or flagged as 'bad'. Spots were considered to be below the background noise if the sum of the median intensities of the two channels was less than twice the highest mean background of the chip. The chips were normalized by the print-tip Lowess method (Dudoit et al., 2000). The ratio of the mean median intensity of Cy5 over the mean median intensity of $\mathrm{Cy} 3$ was determined for each spot and the fold change values were calculated. A one-class SAM analysis (Tusher et al., 2001) was performed with the MEV software (Saeed et al., 2003) to find genes with changes that occurred consistently in all replicates. A median FDR (false discovery rate) of zero, delta values ranging from 3.14 to 3.63 and a mean change of at least twofold were considered our cut-off for significance.

\section{RESULTS}

\section{Gene expression in an mpraB mutant}

To evaluate the role of MprAB in gene regulation, we generated the H37Rv deletion mutant Rv-D981, which is missing a $1.1 \mathrm{kbp}$ region encoding the predicted DNAbinding domain of MprA, as well as the transmembrane region and part of the kinase domain of MprB (Fig. 1a). Exponential growth rates of Rv-D981 and H37Rv were similar under standard growth conditions in broth (data not shown). The expression of mprA was compared in H37Rv and Rv-D981, by real-time PCR, using a probe and primers that hybridize to a section remaining at the $5^{\prime}$ end of $m p r A$ (Fig. 1a). mprA was highly upregulated in H37Rv by SDS treatment, but in Rv-D981, no induction was detected and basal expression levels were also lower than in H37Rv (Fig. 1b). In the complemented strain, Rv-D981C, which was generated by inserting mprAB into the genome under the control of the mprA promoter, SDS activation of mprA was restored, and basal expression levels were increased compared to Rv-D981 (Fig. 1b). mprA expression in RvD981C was slightly higher than in H37Rv, possibly due to
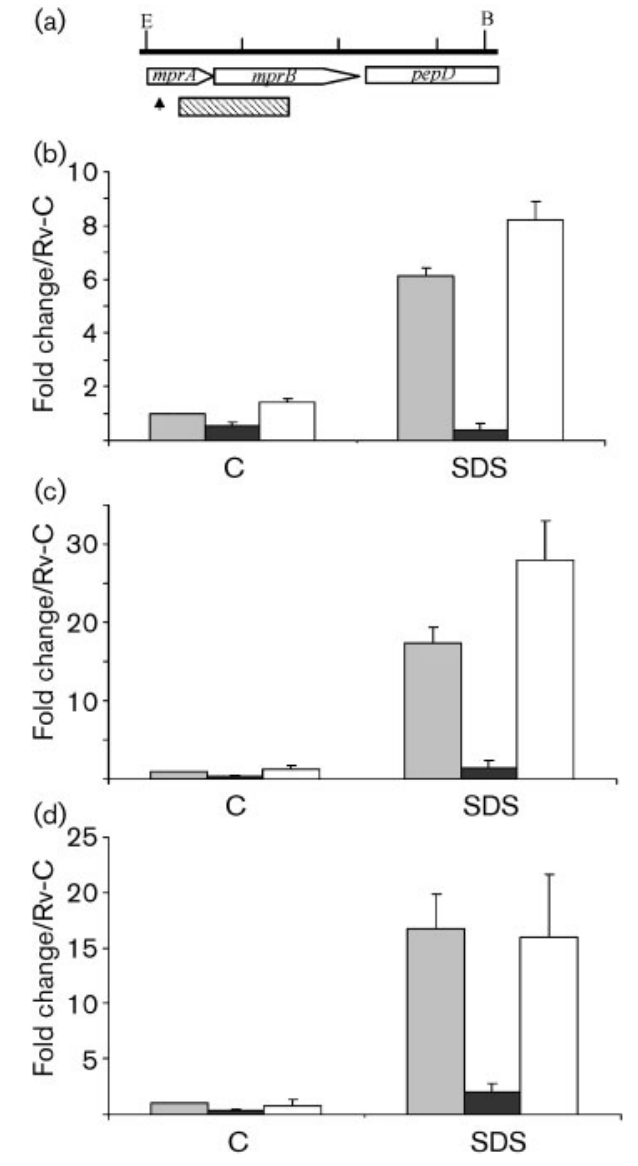

Fig. 1. Impact of Rv-D981 deletion on gene expression. (a) Map of the H37Rv MprAB region and location of Rv-D981 deletion (hatched bar). A portion of $p e p D$ is included. The arrowhead marks the location of $m p r A$ primers and probe used in real-time PCR. E and $\mathrm{B}$ mark EcoRl and BamHI sites used in Southern blot verification of the deletion. Vertical lines mark $1 \mathrm{~kb}$ segments. ( $\mathrm{b}-\mathrm{d}$ ) Expression of $\operatorname{mprA}(\mathrm{b}), \operatorname{sig} E$ (c) and $\operatorname{sig} B$ (d) was examined by real-time PCR using RNA from H37Rv (grey bars), Rv-D981 (black bars), and Rv-D981C (white bars). C, control cultures; SDS, SDS-treated cultures. Results were normalized for 16S RNA content and are shown as fold change over the H37Rv control, which was given a value of 1 . Data are the mean $\pm S D$ of three or more separate experiments with H37Rv and Rv-D981, and two or more experiments with Rv-D981C.

differences in the transcriptional environment, but, overall, these results indicated that the MprAB TCS is required for induction of $m p r A$ expression under detergent stress, and that it contributes to mprA expression during normal exponential growth.

As MprAB was the only TCS regulated by SigE under SDS stress, we hypothesized that MprAB might contribute to activation of the SDS response. We therefore examined expression of the SDS-responsive sigma factor genes, sigE and $\operatorname{sig} B$, in Rv-D981. Both genes were highly activated by SDS exposure in H37Rv (Fig. 1c, d), but expression levels 
were low in Rv-D981 under SDS or control conditions. In the complemented strain, sigE and $\operatorname{sig} B$ were again inducible by SDS (Fig. 1c, d). These data indicate that under the conditions tested, MprAB directly or indirectly regulated the expression of two key sigma factors.

\section{Interaction of MprA with the mprA promoter}

To determine whether MprA influences transcript initiation under SDS stress, we first identified the mprA transcriptional start point (TSP), using primer extension analysis. Preliminary analyses with two primers identified a single TSP located close to the coding region (data not shown). A third primer, 981PE3, was used to verify these results (Fig. 2a). The TSP is a guanosine residue four bases downstream of the predicted MprA start codon (Fig. 2a, b), indicating that mprA is shorter than predicted (Cole et al., 1998). The TSP is also 22 bases downstream of the end of the previously identified MprA-binding sites ( $\mathrm{He} \&$ Zahrt, 2005). The position of the TSP indicates that the -35 region of the mprA promoter overlaps the first direct repeat of the MprA-binding site. The sequence GGCCA near the end of this site (Fig. 2b) has some similarity to the consensus sequences for SigE- and SigH-dependent promoters (Manganelli et al., 2001, 2002; Raman et al., 2001), consistent with the regulation of mprA by SigE (Manganelli et al., 2001). It is likely, however, that, when MprA is bound, sigma factors do not interact directly with this -35 region. The 'GTT' core of the SigE/SigH consensus -10 region (Manganelli et al., 2001, 2002; Raman et al., 2001) was not detected in the MprA promoter.

Importantly, the same TSP was identified in $\mathrm{H} 37 \mathrm{Rv}$ and RvD981, under SDS treatment and control conditions (Fig. 2c). However, mprA transcripts were only weakly

(a)

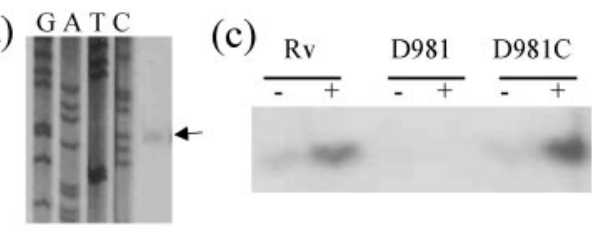

(b)

\section{-41 CTCTCAGGCCAGTCTCAGGCGCTGCGA -14 CGACACTG $\underline{\text { GTGTCC*GTGCGAATTCTT }}$}

Fig. 2. Transcriptional analyses of the $m p r A$ promoter. (a) The mprA TSP (arrow) was mapped by primer extension using H37Rv RNA and primer 981PE3. Sequence ladder was generated using 981PE3. (b) Sequence of the mprA promoter beginning 41 bases upstream of the TSP ( $G$ with asterisk). MprA-binding motifs (He \& Zahrt, 2005) (underlined) and the predicted GTG start codon (underlined, bold) are marked. (c) Primer extension results using primer 981PE3 and RNA from cultures exposed (+) or not exposed $(-)$ to SDS. Rv, H37Rv; D981, Rv-D981; D981C, Rv-D981C. Bands in Rv-D981 were weakly visible and were of similar intensity in each condition. detectable in Rv-D981, compared to H37Rv and Rv-D981C, and were not induced by SDS. These data again indicated that MprA is autoregulatory under SDS stress and control conditions, but showed that it does not alter the site of transcript initiation.

\section{Interaction of MprA with the sigE promoter}

Three TSPs were previously identified for sigE in the $M$. tuberculosis complex (Fig. 3a): TSP1 and TSP2 in M. bovis BCG (Wu et al., 1997), and a SigH-dependent TSP (TSP3) (Raman et al., 2001) located within the predicted coding region of $M$. tuberculosis sigE (Cole et al., 1998). We identified an additional start site, TSP4, which is coincident with the putative start codon of sigE, and upstream of TSP3 (Fig. 3a, b, g), supporting reports that sigE is smaller than predicted (Raman et al., 2001; Wu et al., 1997). TSP4 is activated by SDS stress in H37Rv, in an MprA-dependent manner, as indicated by the reduced intensity of bands for Rv-D981 (Fig. 3b). TSP3 was also activated by SDS stress, but this activation was independent of MprA, as transcription levels were similar in $\mathrm{H} 37 \mathrm{Rv}$ and Rv-D981 (Fig. 3c). MprA may contribute to basal levels of transcription from both TSP3 and TSP4, however, as indicated by the weaker bands in Rv-D981 under control conditions (Fig. 3b, c).

The capacity of MprA to bind the sigE promoter directly was examined by EMSA. MprA shifted the 114 bp sigE promoter probe P1 (Fig. 3d, e), and additional probes (P2-P6) localized MprA binding to within a $54 \mathrm{bp}$ region (P6) (Fig. 3d, and data not shown). Sequences within P6 showed some similarity to the MprA consensus binding site ( $\mathrm{He} \&$ Zahrt, 2005) and were examined using shorter probes (P7P9) (Fig. 3d). P8, which had five bases flanking the putative binding sites, was sufficient to obtain binding (Fig. 3d, $\mathrm{f}$, and data not shown). Mutating bases within the first (P10 and P12) or second (P11) predicted MprA-binding sites within P8 inhibited MprA binding (Fig. 3d, f), confirming these sites as targets for MprA.

The location of TSP4 places the -35 region of the sigE promoter shortly downstream of the MprA-binding sites (Fig. 3g). Sequences beginning at -33 (TGGCCCA) and -11 (CGGTA) each share four bases, respectively, with the -35 and -10 regions of SigH-dependent promoters (Manganelli et al., 2002; Raman et al., 2001). However, as some of the invariant residues reported for $\mathrm{SigH}$-dependent promoters are missing (Manganelli et al., 2002; Raman et al., 2001), it is likely that another sigma factor regulates $\operatorname{sig} E$ from this promoter.

\section{Interaction of MprA with the sigB promoter}

Only a single TSP was identified for sigB (Fig. 4a), which corresponds to the site previously reported (Manganelli et al., 2002; Raman et al., 2001). Activation of this TSP under SDS stress was MprA-dependent (Fig. 4a). We detected MprA binding to probes extending $131 \mathrm{bp}$ upstream of the sigB TSP (Fig. 4b, c, and data not shown), and a search of the 
(a)

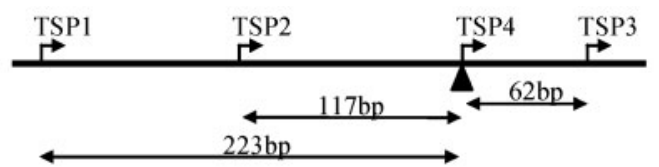

(b)

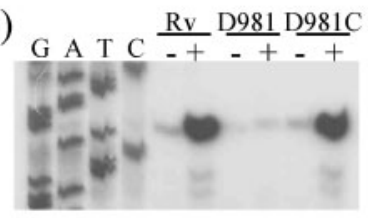

(c)

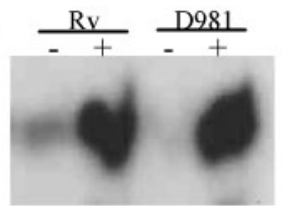

(d) Probe

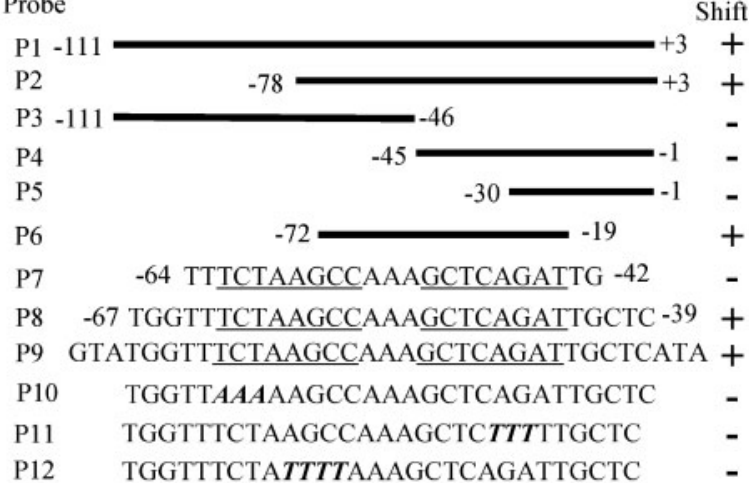

(e)

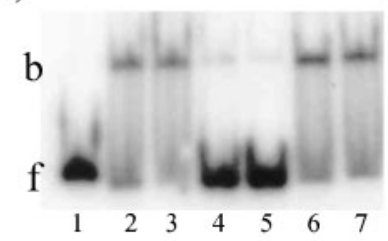

(f) $\frac{\mathrm{P} 8}{-+} \frac{\mathrm{P} 10}{-+} \frac{\mathrm{P} 11}{-+} \frac{\mathrm{P} 12}{-+}$

(g) -62 TCTAAGCCAAAGCTCAGATTGCTCATATATGGCCCA

Fig. 3. Analyses of the sigE promoter. (a) Map of sigE TSPs. Bent arrows mark four identified TSPs (see text for details). Doubleheaded arrows indicate distance in bp between the predicted start codon (vertical arrowhead) and each TSP. TSP4 overlaps the predicted start codon. (b, c) TSP4 (b) and TSP3 (c) were identified by primer extension using primers SigEPE-1 and SigEPE-2, respectively. Sequence ladder was generated with SigEPE-1. (d-f) EMSA analyses with the sigE promoter and MprA. (d) sigE promoter probes P1-P12 were shifted (+) or not shifted (-) by MprA. Numbers indicate position of probes with respect to TSP4. Predicted MprA-binding sites are underlined in P7-P9. Bold italics, bases mutagenized in P10P12. (e) A fixed amount of labelled P1 was incubated in reactions containing: no MprA (lane 1); $0.36 \mu \mathrm{g}$ or $0.72 \mu \mathrm{g} \mathrm{MprA}$ (lanes 2 and 3, respectively); $0.72 \mu \mathrm{g} \mathrm{MprA}$ and 100- or 200fold excess of unlabelled P1 (lanes 4 and 5, respectively); or $0.72 \mu \mathrm{g} \mathrm{MprA}$ and $100-$ or 200 -fold excess of unlabelled control fragment (lanes 6 and 7, respectively). (f) P8-P12 incubated with $(+)$ or without $(-)$ MprA. b, bound probe; $f$, free probe. (g) Map of the sigE promoter for TSP4. Underlined bases indicate MprA-binding sites. Bold bases indicate putative -35 and -10 regions. The start codon is in italics and the asterisk indicates the TSP. (a)

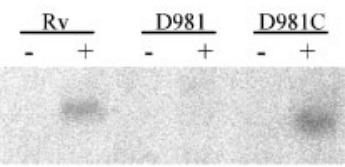

(b)
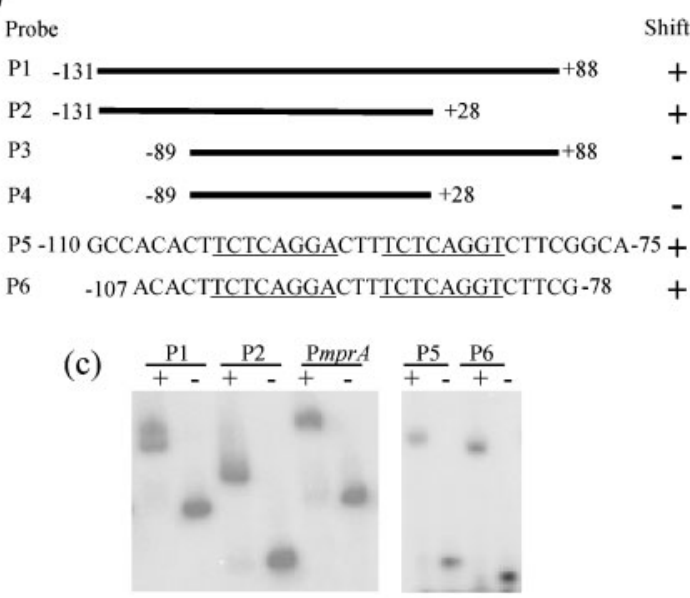

(d)

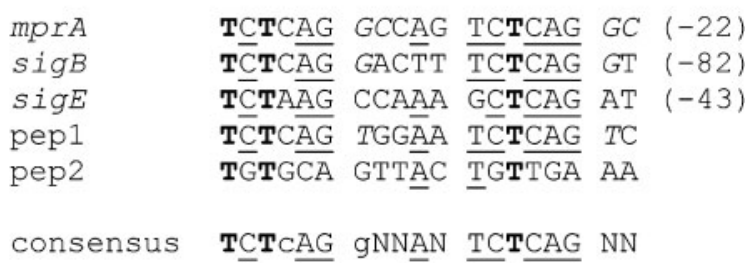

Fig. 4. Analyses of the $\operatorname{sig} B$ promoter and comparison of MprA-binding sites. (a) The sigB TSP was mapped by primer extension using primer SigBPE-1. The D981C band is lower due to curvature of the gel. $(b, c)$ EMSAs with $\operatorname{sig} B$ promoter probes and MprA. (b) Probes P1-P6 were shifted (+) or not shifted ( - ) by MprA. Probe location is indicated with respect to the sigB TSP (Manganelli et al., 2002; Raman et al., 2001). Predicted MprA-binding sites are underlined. (c) Probes P1, P2, P5 and P6 were incubated with $(+)$ or without $(-)$ MprA. PmprA, mprA promoter probe used as positive control. (d) Alignment of MprA-binding sites. Two highly conserved $6 \mathrm{bp}$ motifs are separated by $5 \mathrm{bp}$ spacer. Numbers indicate the position of the last base shown with respect to the TSP: mprA TSP, this study; sigB TSP, Manganelli et al. (2002), Raman et al. (2001); sigE, TSP4, this study. mprA and pepD (pep1, pep2) sites were obtained from published data (He \& Zahrt, 2005). A very weak MprA-binding site in the pepD promoter (He \& Zahrt, 2005) was excluded from the comparison. Positions conserved in three, four or five sequences are indicated by small, underlined or bold letters, respectively. N, any nucleotide. Italics indicate extended direct repeats.

Tuberculist database with the published MprA-binding sequence (He \& Zahrt, 2005) identified potential MprA target sites between positions -102 and -83 . Similar to the findings with the sigE promoter, a $\operatorname{sig} B$ promoter probe (P6) with five bases flanking these potential target sites was sufficient to obtain binding with MprA (Fig. 4b, c). 
Alignment of MprA-binding sites identified by us and by others (He \& Zahrt, 2005) suggests that MprA binds to a core sequence of six highly conserved nucleotides (TCTCAG), separated from a direct repeat by five less-conserved or non-conserved residues (Fig. 4d). A search of the H37Rv genome with this repeat sequence, allowing for up to two mismatches per repeat, revealed 17 other potential sites in intergenic regions (Supplementary Table S2), suggesting that MprA may directly regulate additional genes.

\section{Global patterns of gene expression in Rv-D981}

As our data indicated that MprA activates sigE, we expected that the global gene expression profile of Rv-D981 under SDS stress would be similar to that described for the $\operatorname{sig} E$ deletion mutant (Manganelli et al., 2001). DNA microarray analyses were used to compare gene expression in $\mathrm{H} 37 \mathrm{Rv}$ and Rv-D981, under control conditions and during SDS stress. SDS exposure induced marked changes in gene expression, upregulating and downregulating over 200 genes in both H37Rv and Rv-D981 (Supplementary Tables S3 and S4). Compared to Rv-D981, 39 genes were significantly overexpressed in $\mathrm{H} 37 \mathrm{Rv}$ in SDS, whereas 44 genes were more highly upregulated in Rv-D981 (Table S3), indicating that MprA has both positive and negative effects on gene expression patterns. We examined the expression patterns of the genes with potential MprA-binding sites (Table S2), but did not detect any overall similarity in their expression profiles in Rv-D981 (Table S4 and data not shown).

Based on their reduced expression in the sigE mutant under SDS stress, 23 genes were reported to be directly or indirectly regulated by SigE (Manganelli et al., 2001). DNA microarray analyses showed that ten of these genes were markedly downregulated in Rv-D981 under SDS stress compared to H37Rv (Table 1), and this was confirmed for several genes by real-time PCR (Table 1 and data not shown). Significant changes in expression levels of the SigE-regulated genes $m p r A$, $m p r B$ and $p e p D$ could not be detected by DNA microarray analyses, perhaps due to low expression levels, but real-time PCR confirmed that expression of all three genes was reduced in Rv-D981 (Fig. 1b, Table 1, and data not shown).

Unexpectedly, Rv1129c, Rv1130 and Rv1131, which were downregulated in the sigE mutant under SDS stress (Manganelli et al., 2001), were upregulated in Rv-D981 (Table 1). Preliminary analyses suggested that expression of these genes in Rv-D981 was influenced by the atmospheric conditions used during culture (data not shown). To analyse this more closely, we performed real-time PCR on Rv1129c, using cultures grown for several weeks either with $5 \% \mathrm{CO}_{2}$

Table 1. Expression patterns of SigE-regulated genes in H37Rv and Rv-D981 under SDS stress

The expression of SigE-regulated genes (Manganelli et al., 2004) was evaluated in SDS-treated and control cultures of H37Rv and Rv-D981, using array analyses or real-time PCR (qPCR). For each gene, results show fold induction under SDS compared to the control sample for the same strain.

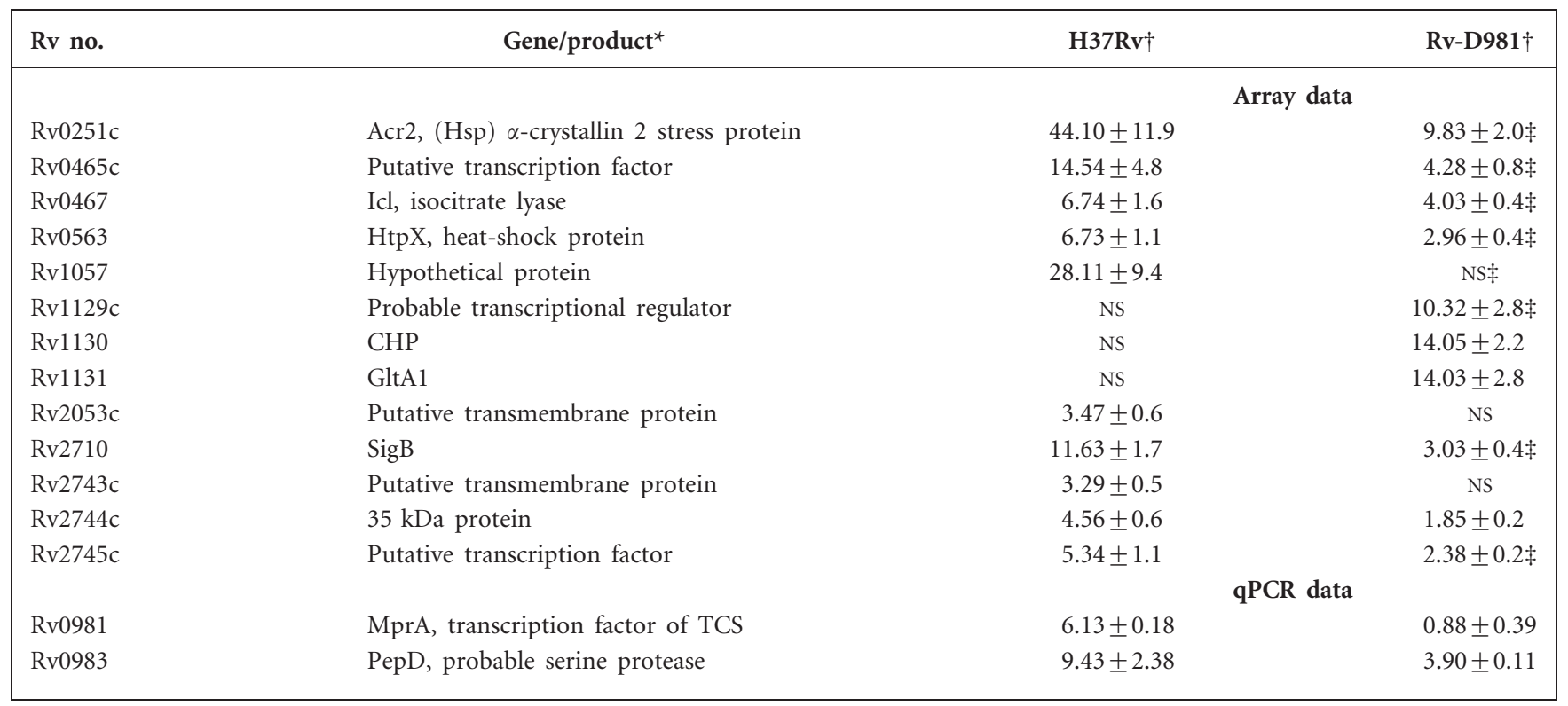

${ }^{\star}$ From http://genolist.pasteur.fr/TubercuList/; CHP, conserved hypothetical protein.

$\dagger$ Data show mean fold induction ( \pm SEM), determined by microarrays (Table S4) or real-time PCR analyses, using three different RNA preparations. NS, No significant increase in expression under SDS compared to control as determined by one-class SAM analysis (Tusher et al., 2001).

‡Differences in gene expression patterns between H37Rv and Rv-D981 were confirmed by real-time PCR. 
or with normal atmospheric $\mathrm{CO}_{2}$. Following SDS stress, Rv1129c was slightly induced in H37Rv, independent of the culture conditions (Fig. 5). However, in Rv-D981, Rv1129c expression was markedly increased by SDS stress, but only in cultures grown under normal atmospheric conditions (Fig. 5). We used EMSA to determine whether MprA directly regulates Rv1129c and several other genes of the SigE regulon, including Rv0465, Rv0467, Rv1057 and Rv2745, but did not detect binding of MprA to these promoters (data not shown), indicating that the effects of MprA are indirect.

\section{Survival phenotype of Rv-D981}

The sigE mutant had lower survival under SDS stress and had reduced growth in macrophages, compared to H37Rv (Manganelli et al., 2001), so we examined survival of RvD981 under these conditions. Broth cultures were exposed to $0.05 \%$ SDS, and percentage survival was determined by comparison of c.f.u. with untreated controls for each strain. All strains had reduced survival after $2 \mathrm{~h}$ exposure to SDS (data not shown), but Rv-D981 had consistently higher survival rates compared to H37Rv and Rv-D981C (Fig. 6a). Preliminary analyses indicate that Rv-D981 also has increased survival in broth in the presence of deoxycholate (G. Mukamolova, unpublished data).

An mprA insertion mutant of $M$. tuberculosis had higher replication rates than $\mathrm{H} 37 \mathrm{Rv}$ in murine macrophages (Zahrt \& Deretic, 2001). To investigate growth of the mprAB deletion mutant in human monocytes, we used a lowinoculum assay (Byrd, 1997), and bacterial growth was evaluated over time (Fig. 6b). Rv-D981 multiplied to a significantly greater extent than $\mathrm{H} 37 \mathrm{Rv}$ by 4 days postinfection, and continued to grow at significantly higher levels by day 7 . Rv-D981C had growth rates similar to

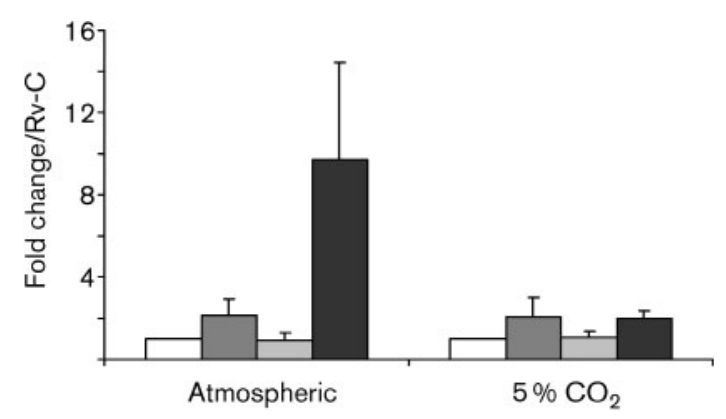

Fig. 5. Expression pattern of Rv1129c under different growth conditions. Cultures of H37Rv and Rv-D981 were grown for several weeks at $37^{\circ} \mathrm{C}$ under normal atmospheric conditions or with $5 \% \mathrm{CO}_{2}$. Aliquots were treated with $0.05 \%$ SDS for 90 min or left untreated and then RNA was extracted and examined by real-time PCR. Results show the mean $\pm S D$ of triplicate experiments. White bars, H37Rv control; dark grey bars, H37Rv with SDS; light grey bars, Rv-D981 control; black bars, Rv-D981 with SDS. (a)
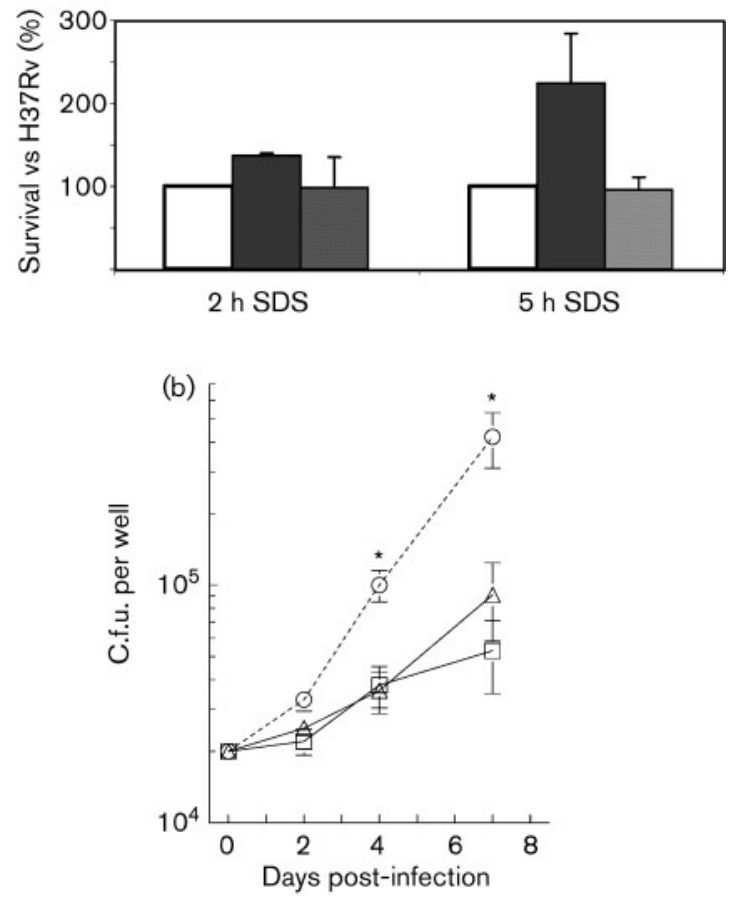

Fig. 6. Rv-D981 has increased stress resistance. (a) Cultures were incubated with $0.05 \%$ SDS and aliquots were removed after $2 \mathrm{~h}$ or $5 \mathrm{~h}$ incubation, washed, and plated in duplicate. Control (untreated) aliquots were plated at time zero. C.f.u. were determined after 14 days. Due to variablility in the absolute number of c.f.u. between experiments, data were calculated as percentage survival compared to untreated controls for each strain. The percentage survival of $\mathrm{H} 37 \mathrm{Rv}$ at each time point was given a value of $100 \%$ and this was compared to the percentage survival of the other two strains. H37Rv, white bars; Rv-D981, dark grey bars; Rv-D981C, light grey bars. Results show the mean \pm SEM from three separate experiments for H37Rv and Rv-D981, and two experiments for Rv-D981C. (b) Growth of Rv-D981 is increased in human monocytes. Human monocyte monolayers were infected with a bacteria: monocyte ratio of $0.2: 1$. At 2,4 and 7 days, c.f.u. in supernates and monocyte lysates were determined. C.f.u. in the supernates and lysates were assayed individually and values were added together to give total c.f.u. per well at each time point (Byrd, 1997). Data are mean \pm SEM for duplicate determinations in three separate experiments using monocytes from different donors. *, statistically significant difference $(P<0.05)$ in growth of Rv-D981 compared to H37Rv and Rv-D981C at 4 and 7 days. $\triangle$, H37Rv; $\bigcirc$, Rv-D981; $\square$, Rv-D981C.

that of H37Rv (Fig. 6b), indicating that loss of mprAB was responsible for the enhanced growth of Rv-D981.

\section{Overexpression of stress-associated genes in Rv-D981}

The basis for the increased survival of Rv-D981 under stress is not clear. However, using the MtbReglist database 
(Jacques et al., 2005), we examined the regulatory features of several genes that were upregulated in Rv-D981 under normal growth conditions, and noted that many had either DosR- or SigD-binding sites in their upstream regions (Table 2). Further comparisons with published data (Kendall et al., 2004; Park et al., 2003; Raman et al., 2004) revealed that, of the 30 most-highly expressed genes in Rv-D981, 21 are members of the DosR or SigD regulons (Table 2). Another five genes are adjacent to members of these regulons and may be at least partially regulated by SigD or DosR. The DosR and SigD regulons are associated with growth under stress (Betts et al., 2002; Calamita et al., 2005; Park et al., 2003; Raman et al., 2004; Sherman et al., 2001), and as discussed below, the derepression of some of these genes in Rv-D981 may contribute to the resistance phenotype we observed. We performed gel-shift assays with MprA and promoters of several genes from these regulons, including Rv1738, Rv3131, Rv2626c and Rv3134c, but did not detect binding (data not

Table 2. Genes highly upregulated in exponential phase in Rv-D981 compared to H37Rv

Expression under control conditions was compared using DNA microarray analyses. The 30 genes with the highest mean fold increase in expression in Rv-D981 compared to H37Rv are shown.

\begin{tabular}{|c|c|c|c|}
\hline Rv no. & Gene/product ${ }^{*}$ & Mean fold increase $\dagger$ & Regulatory features $\ddagger$ \\
\hline Rv3131 & $\mathrm{CHP}$ & $21.34 \pm 6.0$ & $\mathrm{R}$ \\
\hline Rv1738 & $\mathrm{CHP}$ & $15.56 \pm 5.0$ & $\mathrm{R}+$ \\
\hline Rv3130c & $\mathrm{CHP}$ & $15.15 \pm 3.6$ & $\mathrm{R}$ \\
\hline Rv1996 & USP & $9.91 \pm 1.3$ & $\mathrm{R}$ \\
\hline Rv3127 & $\mathrm{CHP}$ & $8.98 \pm 1.7$ & $\mathrm{R}+$ \\
\hline Rv1884c & RpfC, resuscitation-promoting factor & $5.95 \pm 0.6$ & $\mathrm{D}+$ \\
\hline Rv2032 & Acg & $5.55 \pm 1.3$ & $\mathrm{R}+$ \\
\hline Rv2626c & $\mathrm{CHP}$ & $5.24 \pm 0.8$ & $\mathrm{R}+$ \\
\hline Rv2627c & $\mathrm{CHP}$ & $4.81 \pm 1.4$ & $\mathrm{R}+$ \\
\hline Rv0079 & Hypothetical protein & $4.16 \pm 0.9$ & $\mathrm{R}+$ \\
\hline Rv1733c & Putative transmembrane protein & $4.11 \pm 0.5$ & $\mathrm{R}+$ \\
\hline Rv1815 & $\mathrm{CHP}$ & $4.04 \pm 0.2$ & $\mathrm{D}+$ \\
\hline Rv2623 & USP & $3.91 \pm 0.4$ & $\mathrm{R}$ \\
\hline Rv3134c & USP & $3.69 \pm 0.6$ & $\mathrm{R}+$ \\
\hline Rv0569 & $\mathrm{CHP}$ & $3.43 \pm 0.5$ & $\mathrm{R}$ \\
\hline Rv2628 & $\mathrm{CHP}$ & $3.25 \pm 0.7$ & $\mathrm{R}+$ \\
\hline $\operatorname{Rv} 3229 c$ & Putative linoleoyl-CoA desaturase & $3.10 \pm 0.4$ & \\
\hline Rv1737c & NarK2, nitrate/nitrite transporter & $3.09 \pm 0.7$ & $\mathrm{R}+$ \\
\hline Rv2557 & $\mathrm{CHP}$ & $3.09 \pm 0.4$ & \\
\hline Rv2632c & $\mathrm{CHP}$ & $3.06 \pm 0.3$ & (D), (R) \\
\hline Rv2007c & FdxA, probable ferredoxin & $2.92 \pm 0.5$ & $\mathrm{R}+$ \\
\hline Rv3616c & $\mathrm{CHP}$ & $2.92 \pm 0.2$ & (D) \\
\hline Rv1181 & Pks4, polyketide synthase, (msl3) & $2.81 \pm 0.3$ & (D) \\
\hline $\operatorname{Rv} 3083$ & Probable monooxygenase & $2.78 \pm 0.2$ & \\
\hline Rv2160c & CHP & $2.69 \pm 0.2$ & \\
\hline Rv1883c & $\mathrm{CHP}$ & $2.66 \pm 0.3$ & $\mathrm{D}$ \\
\hline Rv1813c & $\mathrm{CHP}$ & $2.63 \pm 0.2$ & $\mathrm{R}+$ \\
\hline Rv3614c & $\mathrm{CHP}$ & $2.47 \pm 0.3$ & $\mathrm{D}$ \\
\hline Rv1183 & MmpL10, fatty acid transport (putative) & $2.38 \pm 0.1$ & (D) \\
\hline Rv1814 & Sterol desaturase & $2.38 \pm 0.2$ & (D), (R) \\
\hline
\end{tabular}

${ }^{*}$ From http://genolist.pasteur.fr/TubercuList/; msl3 (Dubey et al., 2002); CHP, conserved hypothetical protein; USP, universal stress protein genes (O’Toole \& Williams, 2003).

$\dagger$ Results show mean fold induction $( \pm$ SEM) of genes with the highest level of expression in Rv-D981 compared to H37Rv, as determined by one-class SAM analysis (Tusher et al., 2001) (Table S4). Only genes showing significant increase in $\geqslant 5$ hybridizations (Table S4), and which are present in the H37Rv strain of M. tuberculosis, are included.

$\ddagger \mathrm{R}$ or $\mathrm{D}$, member of the DosR or SigD regulon, respectively; +, DosR- or SigD-binding site in promoter; (D) or (R), adjacent to gene of the DosR or SigD regulon, respectively. Data obtained from published reports (Raman et al., 2004; Betts et al., 2002; Jacques et al., 2005; Kendall et al., 2004; Park et al., 2003; Sherman et al., 2001). 
Table 3. Expression of iron-responsive genes in H37Rv and Rv-D981 under SDS stress

The expression of iron-regulated genes was evaluated in SDS-treated and control cultures of H37Rv and Rv-D981, using DNA microarray analyses. For each gene, results show fold induction under SDS compared to the control sample for the same strain.

\begin{tabular}{|lccc|}
\hline Rv no. & Gene/product $^{*}$ & H37Rv $\dagger$ & Rv-D981 $\dagger$ \\
\hline Rv2711 & ideR & $3.47 \pm 0.3$ & NS \\
Rv2122c & hisE $($ irg1 & NS & $5.21 \pm 1.0$ \\
Rv2123 & PPE & NS & $5.03 \pm 1.0$ \\
Rv2381c & $m b t D$ & $2.70 \pm 0.1$ & $4.03 \pm 0.6$ \\
Rv2383c & mbtB & $4.74 \pm 0.6$ & $7.28 \pm 1.4$ \\
Rv3839 & CHP & NS & $2.00 \pm 0.1$ \\
\hline
\end{tabular}

*From http://genolist.pasteur.fr/TubercuList/. Published reports (De Voss et al., 1999; Dussurget et al., 1999; Gold et al., 2001; Jacques et al., 2005; Manabe et al., 1999; Prakash et al., 2005) were used to identify genes regulated, or predicted to be regulated, by iron and IdeR. CHP, conserved hypothetical protein.

$\dagger$ Data show mean fold induction ( \pm SEM), determined by DNA microarray analyses (Table S4), using three different RNA preparations and dye flips. NS, No significant increase in expression under SDS compared to control, as determined by one-class SAM analysis (Tusher et al., 2001).

shown), suggesting that the inhibitory effects of MprA on many of these genes is indirect.

We also noted that, under SDS stress, the iron-repressor gene ideR (Dussurget et al., 1999; Gold et al., 2001) was downregulated in Rv-D981 (Table 3 and Table S4). Therefore, we examined expression of genes known to be repressed by IdeR or with potential IdeR-binding sites in their promoters (Dussurget et al., 1999; Gold et al., 2001; Jacques et al., 2005; Manabe et al., 1999; Prakash et al., 2005). Five such genes, including the mycobactin synthetase genes $m b t B$ and $m b t D$ (De Voss et al., 1999), were more highly upregulated in Rv-D981 than in H37Rv under SDS stress (Table 3 ).

\section{DISCUSSION}

The controlled expression of regulatory networks and metabolic systems is necessary for bacteria to adapt efficiently to changing environmental conditions. Our investigations have shown that the MprAB TCS activates the SigE regulon under SDS stress. However, our results also indicate that, in the absence of MprAB, many stressassociated genes are upregulated under normal growth conditions, suggesting that the MprA regulon contributes to maintaining a balance among several systems involved in stress resistance.

In this work, we constructed a deletion mutant, Rv-D981, which lacks portions of $m p r A$ and $m p r B$, thus confirming that both genes are nonessential. We then determined, by real-time PCR, DNA microarrays, and primer extension analyses, that MprAB activates sigE, sigB and mprA under SDS stress, and contributes to maintaining basal expression levels of these genes during exponential growth. Normal patterns of expression were restored in the complemented strain, Rv-D981C, confirming that deletion of $m p r A$ and $m p r B$ was responsible for the changes in transcription patterns. We also identified the TSP for mprA, as well as a new MprA-inducible TSP for sigE, and showed that, under SDS stress, MprA induces transcription from the same TSPs as are used at low levels under normal growth conditions. EMSAs with recombinant MprA showed direct interaction with the $\operatorname{sig} E, \operatorname{sig} B$ and $m p r A$ promoters, and determined that a fragment of 29 bp containing MprA-binding sites was of sufficient length for MprA binding. Other potential MprA-binding sites were identified in the genome, suggesting that MprA may directly regulate additional genes. DNA microarrays showed that many genes of the SigE regulon (Manganelli et al., 2001) were downregulated in the MprAB mutant, consistent with activation of $s i g E$ by MprA. Notable exceptions were the genes Rv1129c-Rv1130, which were more highly upregulated under SDS stress in Rv-D981 than in H37Rv, but only in normal atmospheric conditions.

As summarized in Fig. 7, components of several stressassociated regulons are upregulated in Rv-D981. Although the basis for this upregulation is unknown, the MprA regulon may have a repressive effect on some stress regulons, perhaps as a mechanism for keeping a balanced response to environmental stress. Alternatively, these other stressresponse systems may be upregulated to compensate for the loss of a functional MprAB TCS, and reduced expression of the SigE regulon (Table 1). However, in contrast to a sigE deletion mutant (Manganelli et al., 2001), Rv-D981 had increased resistance to SDS and enhanced growth in human monocytes (this study), and, furthermore, an insertion mutant of mprA had increased growth in resting murine macrophages (Zahrt \& Deretic, 2001), findings which suggest that MprAB has functions independent of its role in activating the SigE regulon.

Deletion of some TCSs can result in hypervirulence (Parish et al., 2003). In the case of Rv-D981, we hypothesize that the higher expression levels of a cohort of stress-associated genes may produce physiological changes that allow the mutant to survive better when exposed to certain environmental stresses. For example, the iron-regulated gene, $m b t B$, which is involved in synthesis of the siderophore mycobactin and which is upregulated in Rv-D981 (Table 3), is important for growth under low-iron conditions and in monocytes (De Voss et al., 2000). Also, analyses of gene expression in RvD981 under normal growth conditions (Table 2 and Table S4), revealed that 21 of the most highly upregulated genes are part of either of the DosR (Kendall et al., 2004; Park et al., 2003) or SigD regulons. In contrast, only two of these genes, Rv1884c and Rv3616c, were upregulated by $\geqslant 2$-fold in the sigE mutant during exponential-phase 


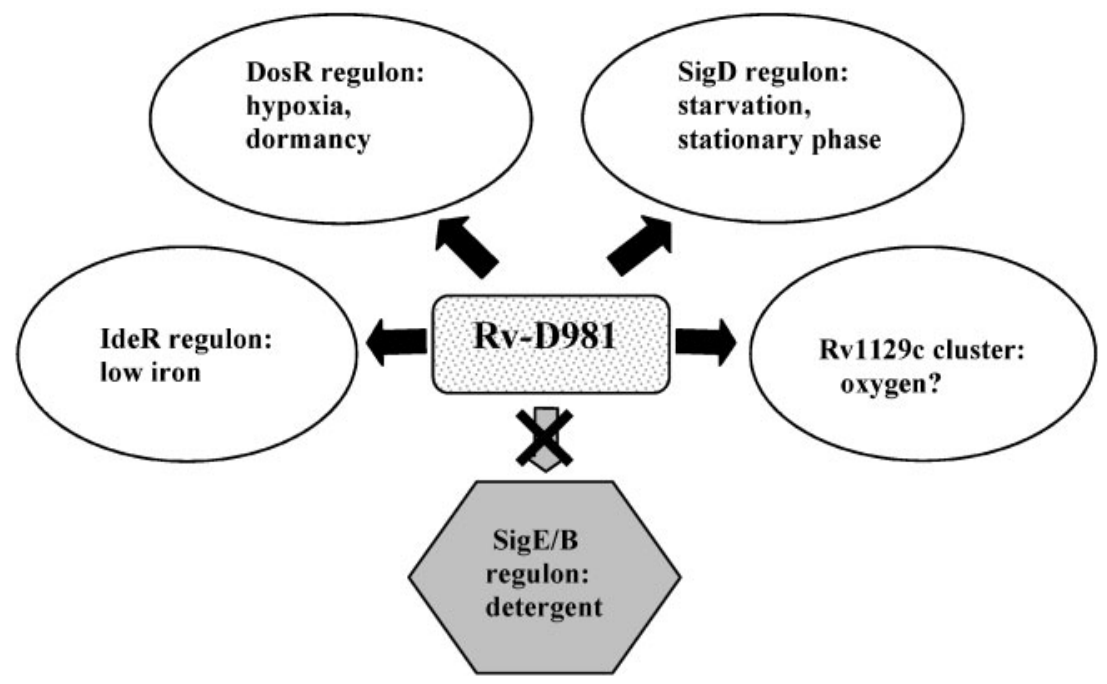

Fig. 7. Schematic diagram showing altered expression pattern of stress-associated regulons in Rv-D981. Ovals with black arrows indicate upregulation, and the hexagon with a crossed arrow indicates downregulation. Stresses associated, or potentially associated, with genes/regulons are indicated.

growth (Manganelli et al., 2001). Several other genes of the SigD and DosR regulons are also more highly expressed in Rv-D981 under SDS stress (Table S4 and data not shown).

Genes of the sigD regulon are associated with nutrient starvation and stationary phase (Betts et al., 2002; Calamita et al., 2005; Raman et al., 2004), and sigD mutants of $M$. tuberculosis are attenuated (Calamita et al., 2005; Raman et al., 2004). Genes of the DosR (also called DevR) regulon are upregulated under hypoxia (Park et al., 2003; Sherman et al., 2001) and other stress conditions (Karakousis et al., 2004; Kendall et al., 2004; Ohno et al., 2003; Voskuil et al., 2003), and contribute to survival under low-oxygen conditions (Boon \& Dick, 2002). Genes of these regulons which could contribute to enhanced survival include those encoding the universal stress proteins, as well as NarK2 and RpfC (Table 2).

Universal stress proteins are associated with various mechanisms of stress resistance (Nachin et al., 2005; O’Toole \& Williams, 2003), including long-term survival of M. tuberculosis under low-oxygen conditions (Voskuil et al., 2003). NarK2 (Rv1737c) is important for nitrate reductase activity (Sohaskey \& Wayne, 2003), and may be involved in adaptation to nitric oxide stress in macrophages (Shi et al., 2005). RpfC is one of five M. tuberculosis proteins with similarity to Rpf (resuscitation-promoting factor) of Micrococcus luteus, which are important for stimulation of growth of $M$. tuberculosis in broth, in recovery of dormant mycobacteria, and in virulence (Downing et al., 2005; Mukamolova et al., 2002; Tufariello et al., 2006). Recent studies suggest that Rpf-like proteins are muralytic enzymes involved in remodelling of the bacterial cell wall (CohenGonsaud et al., 2005; Mukamolova et al., 2006). In addition, several genes involved in fatty acid metabolism are upregulated in Rv-D981 (Table 2 and Table S4), and evidence indicates that two of these genes, Rv1180 and Rv1181, form a single large gene, named msl3, which encodes an enzyme involved in synthesis of lipid components of the cell wall (Dubey et al., 2002). Increased expression of $r p f C, m s l 3$, and other genes in Rv-D981 could potentially induce structural changes in the cell wall that enhance survival when the mutant is exposed to detergents or during growth in monocytes. Although an mprA insertion mutant showed decreased persistence during the late stage of infection in mice (Zahrt \& Deretic, 2001), it is conceivable that the alterations in gene expression patterns in $m p r A$ mutants may provide some benefit early during infection, and in some other stresses, and yet be deleterious during the persistent phase of infection.

The putative transcriptional regulator, Rv1129c (Cole et al., 1998), was also highly upregulated in Rv-D981 under SDS stress, but this upregulation was abrogated in the presence of $5 \% \mathrm{CO}_{2}$. The role of $\mathrm{Rv} 1129 \mathrm{c}$ is unknown, but it may regulate the adjacent genes, Rv1130 and Rv1131, which showed a similar pattern of expression. Interestingly, this cluster of genes is also upregulated in the phagosomes of infected murine macrophages, and Rv1129c and Rv1131 are active in infected mice (Schnappinger et al., 2003). The function of Rv1130 is unknown, but the protein has been detected in phagosomes and may contribute to intracellular survival (Mattow et al., 2006). Rv1131 (gltA1) is predicted to encode citrate synthase, an enzyme of the TCA (Krebs) cycle (Cole et al., 1998). gltA of E. coli is negatively regulated by the ArcAB TCS, and expression of the gene is sensitive to changes in oxygen levels (Shalel-Levanon et al., 2005). Our data suggest that expression of the Rv1129c-Rv1131 gene cluster may also be influenced by oxygen levels, and that, under some conditions, the gene cluster is negatively regulated when $\mathrm{MprAB}$ is intact.

For each of $\operatorname{sigE}$, sigB and $m p r A$, an MprA-inducible TSP was identified in H37Rv, which was also active in Rv-D981, albeit at lower levels. These data suggest that MprA functions by modulating the activity of the RNA holoenzyme at weak, but active, promoters. We found two SDSinducible TSPs for sigE, although only the newly identified TSP4 was dependent on MprA. For sigB, only a single TSP was identified, the location of which was consistent with the 
previously reported site (Manganelli et al., 2002; Raman et al., 2001), thus placing the end of the MprA-binding site approximately 50 bases upstream of the predicted -35 region (Manganelli et al., 2002; Raman et al., 2001). In contrast, the MprA-binding site in the $m p r A$ promoter overlaps the -35 region, and in the sigE promoter, the -35 region for TSP4 is several bases downstream of the MprA-binding site. Therefore, perhaps similar to the catabolite activator protein (CAP) of E. coli (Busby \& Ebright, 1999), MprA may have different mechanisms of interaction with the RNA holoenzyme and/or other proteins. With class 1 CAP-dependent promoters, the CAP-binding site is located upstream of the -35 region, whereas with class 2 promoters, the CAP-binding site overlaps the -35 region (Busby \& Ebright, 1999). Different protein-protein interactions between CAP and the RNA polymerase are involved at these two classes of promoters. However, the interactions between MprA and other regulatory factors remain to be elucidated.

While this paper was in preparation, another group published work on an MprA insertion mutant showing that MprA regulates sigE and sigB (He et al., 2006). Using DNA microarray analyses, they detected significant changes in gene expression in the mutant under detergent stress, and observed that $\operatorname{sig} E$ and $\operatorname{sig} B$ were downregulated in the mutant during exponential growth. They also localized MprA-binding sites in the sigE and $\operatorname{sig} B$ promoters using DNA footprinting, and their data are consistent with our findings, although they detected an additional weak MprAbinding site in the sigE promoter which we would not have detected using our approach. In contrast to our results, they did not detect a reduction in $\operatorname{sig} B$ expression in the mutant under SDS stress, although sigE was downregulated in their analyses as well as in ours. The basis for this discrepancy is not entirely clear, but they normalized their real-time PCR results to sigA expression, which may have resulted in reduced sensitivity, as sigA expression can change under certain conditions (Wu et al., 2004). However, as sigB was downregulated in a sigE mutant under SDS stress (Manganelli et al., 2001), our findings are consistent with earlier studies. It is also possible that, as $m p r B$ was intact in their mutant (He et al., 2006), MprB may activate another response regulator which activates $\operatorname{sig} B$ under SDS stress. Interestingly, three genes of the DosR regulon (Rv2626cRv2628), which were upregulated in our mutant (Table 2), were downregulated in the mprA insertion mutant (He et al., 2006), suggesting that there may be some distinct differences in gene regulation between the two mutants.

Overall, both studies show the importance of MprA in the response to specific stresses. At the outset of our investigations, we had hypothesized that, as the only TCS in the SigE regulon, MprAB may activate the SigE regulon, and this has been supported by our findings. MprAB is also the only TCS within the iVEGI region, which is highly active in vivo models (Jain et al., 2006; Talaat et al., 2004), so we speculate that MprAB may activate iVEGI genes in vivo. However, further investigations are required to elucidate the obviously complex role of this TCS in vivo and under other stress conditions.

\section{ACKNOWLEDGEMENTS}

We thank Peter F. Barnes for support of this work, helpful discussions, and critical reading of the manuscript. We also thank Hassan Safi, Luc Gaudreau and Sebastien Rodrigue for technical suggestions. This research was supported by the Department of Veterans Affairs and a DeSouza Research Award from the American Lung Association (to T.F.B.) and the Margaret E. Byers Cain Chair for Tuberculosis Research (to P. F. Barnes), a Potts Memorial Foundation Grant, UTHCT Research Council Seed Grant, and NIH grant R21 AI06322901 (to S. T. H.).

\section{REFERENCES}

Ando, M., Yoshimatsu, T., Ko, C., Converse, P. J. \& Bishai, W. R. (2003). Deletion of Mycobacterium tuberculosis sigma factor E results in delayed time to death with bacterial persistence in the lungs of aerosol-infected mice. Infect Immun 71, 7170-7172.

Betts, J. C., Lukey, P. T., Robb, L. C., McAdam, R. A. \& Duncan, K. (2002). Evaluation of a nutrient starvation model of Mycobacterium tuberculosis persistence by gene and protein expression profiling. Mol Microbiol 43, 717-731.

Boon, C. \& Dick, T. (2002). Mycobacterium bovis BCG response regulator essential for hypoxic dormancy. J Bacteriol 184, 6760-6767.

Busby, S. \& Ebright, R. H. (1999). Transcription activation by catabolite activator protein (CAP). J Mol Biol 293, 199-213.

Byrd, T. F. (1997). Tumor necrosis factor $\alpha$ (TNF $\alpha$ ) promotes growth of virulent Mycobacterium tuberculosis in human monocytes. Ironmediated growth suppression is correlated with decreased release of TNF- $\alpha$ from iron-treated, infected monocytes. J Clin Invest 99, 2518-2529.

Byrd, T. F. \& Horwitz, M. (1989). Interferon gamma-activated human monocytes downregulate transferrin receptors and inhibit the intracellular multiplication of Legionella pneumophila by limiting the availability of iron. J Clin Invest 83, 1457-1465.

Calamita, H., Ko, C., Tyagi, S., Yoshimatsu, T., Morrison, N. E. \& Bishai, W. R. (2005). The Mycobacterium tuberculosis SigD sigma factor controls the expression of ribosome-associated gene products in stationary phase and is required for full virulence. Cell Microbiol 7, 233-244.

Cohen-Gonsaud, M., Barthe, P., Bagneris, C., Henderson, B., Ward, J., Roumestand, C. \& Keep, N. H. (2005). The structure of a resuscitation-promoting factor domain from Mycobacterium tuberculosis shows homology to lysozymes. Nat Struct Mol Biol 12, 270-273.

Cole, S. T., Brosch, R., Parkhill, J., Garnier, T., Churcher, C., Harris, D., Gordon, S. V., Eiglemeier, K., Gas, S. \& other authors (1998). Deciphering the biology of Mycobacterium tuberculosis from the complete genome sequence. Nature 393, 537-544.

Corbett, L. \& Raviglione, M. C. (2005). Global burden of tuberculosis: past, present and future. In Tuberculosis and the Tubercle Bacilli, pp. 3-12. Edited by S. T. Cole, K. D. Eisenach, D. N. McMurray \& W. R. Jacobs, Jr. Washington, DC: American Society for Microbiology.

De Voss, J. J., Rutter, K., Schroeder, B. G. \& Barry, C. E., 3rd (1999). Iron acquisition and metabolism by mycobacteria. J Bacteriol 181, 4443-4451.

De Voss, J. J., Rutter, K., Schroeder, B. G., Su, H., Zhu, Y. \& Barry, C. E., 3rd (2000). The salicylate-derived mycobactin siderophores of 
Mycobacterium tuberculosis are essential for growth in macrophages. Proc Natl Acad Sci U S A 97, 1252-1257.

Downing, K. J., Mischenko, V. V., Shleeva, M. O., Young, D. I., Young, M., Kaprelyants, A. S., Apt, A. S. \& Mizrahi, V. (2005). Mutants of Mycobacterium tuberculosis lacking three of the five rpflike genes are defective for growth in vivo and for resuscitation in vitro. Infect Immun 73, 3038-3043.

Dubey, V. S., Sirakova, T. D. \& Kolattukudy, P. E. (2002). Disruption of msl3 abolishes the synthesis of mycolipanoic and mycolipenic acids required for polyacyltrehalose synthesis in Mycobacterium tuberculosis $\mathrm{H} 37 \mathrm{Rv}$ and causes cell aggregation. Mol Microbiol 45, 1451-1459.

Dudoit, S., Yang, Y. H., Callow, M. J. \& Speed, T. P. (2000). Statistical methods for identifying differentially expressed genes in replicated cDNA microarray experiments. Technical Report no. 578, Stanford University.

Dussurget, O., Timm, J., Gomez, M., Gold, B., Yu, S., Sabol, S. Z., Holmes, R. K., Jacobs, W. R., Jr \& Smith, I. (1999). Transcriptional control of the iron-responsive $f x b A$ gene by the mycobacterial regulator IdeR. J Bacteriol 181, 3402-3408.

Geiman, D. E., Kaushal, D., Ko, C., Tyagi, S., Manabe, Y. C., Schroeder, B. G., Fleischmann, R. D., Morrsion, N. E., Converse, P. J. \& other authors (2004). Attenuation of late-stage disease in mice infected by the Mycobacterium tuberculosis mutant lacking the SigF alternate sigma factor and identification of SigF-dependent genes by microarray analysis. Infect Immun 72, 1733-1745.

Gold, B., Rodriguez, G. M., Marras, S. A., Pentecost, M. \& Smith, I. (2001). The Mycobacterium tuberculosis IdeR is a dual functional regulator that controls transcription of genes involved in iron acquisition, iron storage and survival in macrophages. Mol Microbiol 42, 851-865.

He, H. \& Zahrt, T. C. (2005). Identification and characterization of a regulatory sequence recognized by Mycobacterium tuberculosis persistence regulator MprA. J Bacteriol 187, 202-212.

He, H., Hovey, R., Kane, J., Singh, V. \& Zahrt, T. C. (2006). MprAB is a stress-responsive two-component system that directly regulates expression of sigma factors $\mathrm{SigB}$ and $\mathrm{SigE}$ in Mycobacterium tuberculosis. J Bacteriol 188, 2134-2143.

Hoch, J. A. \& Varughese, K. I. (2001). Keeping signals straight in phosphorelay signal transduction. J Bacteriol 183, 4941-4949.

Jacques, P. E., Gervais, A. L., Cantin, M., Lucier, J. F., Dallaire, G., Drouin, G., Gaudreau, L., Goulet, J. \& Brzezinski, R. (2005). MtbRegList, a database dedicated to the analysis of transcriptional regulation in Mycobacterium tuberculosis. Bioinformatics 21, 2563-2565.

Jain, S. K., Paul-Satyaseela, M., Lamichhane, G., Kim, K. S. \& Bishai, W. R. (2006). Mycobacterium tuberculosis invasion and traversal across an in vitro human blood-brain barrier as a pathogenic mechanism for central nervous system tuberculosis. J Infect Dis 193, 1287-1295.

Jensen-Cain, D. M. \& Quinn, F. D. (2001). Differential expression of sigE by Mycobacterium tuberculosis during intracellular growth. Microb Pathog 30, 271-278.

Karakousis, P. C., Yoshimatsu, T., Lamichhane, G., Woolwine, S. C., Nuermberger, E. L., Grosset, J. \& Bishai, W. R. (2004). Dormancy phenotype displayed by extracellular Mycobacterium tuberculosis within artificial granulomas in mice. J Exp Med 200, 647-657.

Kendall, S. L., Movahedzadeh, F., Rison, S. C., Wernisch, L., Parish, T., Duncan, K., Betts, J. C. \& Stoker, N. G. (2004). The Mycobacterium tuberculosis dosRS two-component system is induced by multiple stresses. Tuberculosis 84, 247-255.

Manabe, Y. C., Saviola, B. J., Sun, L., Murphy, J. R. \& Bishai, W. R. (1999). Attenuation of virulence in Mycobacterium tuberculosis expressing a constitutively active iron repressor. Proc Natl Acad Sci U S A 96, 12844-12848.

Manabe, Y. C., Hatem, C. L., Kesavan, A. K., Durack, J. \& Murphy, J. R. (2005). Both Corynebacterium diphtheriae DtxR(E175K) and Mycobacterium tuberculosis $\operatorname{IdeR}(\mathrm{D} 177 \mathrm{~K})$ are dominant positive repressors of IdeR-regulated genes in M. tuberculosis. Infect Immun 73, 5988-5994.

Manganelli, R., Dubnau, E., Tyagi, S., Kramer, F. R. \& Smith, I. (1999). Differential expression of 10 sigma factor genes in Mycobacterium tuberculosis. Mol Microbiol 31, 715-724.

Manganelli, R., Voskuil, M. I., Schoolnik, G. K. \& Smith, I. (2001). The Mycobacterium tuberculosis ECF sigma factor $\sigma^{\mathrm{E}}$ : role in global gene expression and survival in macrophages. Mol Microbiol 41, 423-437.

Manganelli, R., Voskuil, M. I., Schoolnik, G. K., Dubnau, E., Gomez, M. \& Smith, I. (2002). Role of the extracytoplasmic-function $\sigma$ factor $\sigma^{\mathrm{H}}$ in Mycobacterium tuberculosis global gene expression. Mol Microbiol 45, 365-374.

Manganelli, R., Fattorini, L., Tan, D., Iona, E., Orefici, G., Altavilla, G., Cusatelli, P. \& Smith, I. (2004). The extra cytoplasmic function sigma factor $\sigma^{\mathrm{E}}$ is essential for Mycobacterium tuberculosis virulence in mice. Infect Immun 72, 3038-3041.

Mattow, J., Siejak, F., Hagens, K., Becher, D., Albrecht, D., Krah, A., Schmidt, F., Jungblut, P. R., Kaufmann, S. H. \& Schaible, U. E. (2006). Proteins unique to intraphagosomally grown Mycobacterium tuberculosis. Proteomics 6, 2485-2494.

Mecsas, J., Rouviere, P. E., Erickson, J. W., Donohue, T. J. \& Gross, C. A. (1993). The activity of sigma E, an Escherichia coli heatinducible sigma-factor, is modulated by expression of outer membrane proteins. Genes Dev 7, 2618-2628.

Mukamolova, G. V., Turapov, O. A., Young, D. I., Kaprelyants, A. S., Kell, D. B. \& Young, M. (2002). A family of autocrine growth factors in Mycobacterium tuberculosis. Mol Microbiol 46, 623-635.

Mukamolova, G. V., Murzin, A. G., Salina, E. G., Demina, G. R., Kell, D. B., Kaprelyants, A. S. \& Young, M. (2006). Muralytic activity of Micrococcus luteus Rpf and its relationship to physiological activity in promoting bacterial growth and resuscitation. Mol Microbiol 59, 84-98.

Nachin, L., Nannmark, U. \& Nystrom, T. (2005). Differential roles of the universal stress proteins of Escherichia coli in oxidative stress resistance, adhesion, and motility. J Bacteriol 187, 6265-6272.

Ohno, H., Zhu, G., Mohan, V. P., Chu, D., Kohno, S., Jacobs, W. R., Jr \& Chan, J. (2003). The effects of reactive nitrogen intermediates on gene expression in Mycobacterium tuberculosis. Cell Microbiol 5, 637-648.

O’Toole, R. \& Williams, H. D. (2003). Universal stress proteins and Mycobacterium tuberculosis. Res Microbiol 154, 387-392.

O’Toole, R., Smeulders, M. J., Blokpoel, M. C., Kay, E. J., Lougheed, K. \& Williams, H. D. (2003). A two-component regulator of universal stress protein expression and adaptation to oxygen starvation in Mycobacterium smegmatis. J Bacteriol 185, 1543-1554.

Parish, T. \& Stoker, N. G. (2000). Use of a flexible cassette method to generate a double unmarked Mycobacterium tuberculosis tlyA plcABC mutant by gene replacement. Microbiology 146, 1969-1975.

Parish, T., Smith, D. A., Kendall, S., Casali, N., Bancroft, G. J. \& Stoker, N. G. (2003). Deletion of two-component regulatory systems increases the virulence of Mycobacterium tuberculosis. Infect Immun 71, 1134-1140.

Park, H. D., Guinn, K. M., Harrell, M. I., Liao, R., Voskuil, M. I., Tompa, M., Schoolnik, G. K. \& Sherman, D. R. (2003). Rv3133c/dosR is a transcription factor that mediates the hypoxic response of Mycobacterium tuberculosis. Mol Microbiol 48, 833-843. 
Perez, E., Samper, S., Bordas, Y., Guilhot, C., Gicquel, B. \& Martin, C. (2001). An essential role for phoP in Mycobacterium tuberculosis virulence. Mol Microbiol 41, 179-187.

Prakash, P., Pathak, N. \& Hasnain, S. E. (2005). pheA (Rv3838c) of Mycobacterium tuberculosis encodes an allosterically regulated monofunctional prephenate dehydratase that requires both catalytic and regulatory domains for optimum activity. J Biol Chem 280, 20666-20671.

Raman, S., Song, T., Puyang, X., Bardarov, S., Jacobs, W. R., Jr \& Husson, R. N. (2001). The alternative sigma factor SigH regulates major components of oxidative and heat stress responses in Mycobacterium tuberculosis. J Bacteriol 183, 6119-6125.

Raman, S., Hazra, R., Dascher, C. C. \& Husson, R. N. (2004). Transcription regulation by the Mycobacterium tuberculosis alternative sigma factor SigD and its role in virulence. J Bacteriol 186, 6605-6616.

Rison, S. C. G., Kendall, S. L., Movahedzadeh, F. \& Stoker, N. G. (2005). The mycobacterial two-component regulatory systems. In Mycobacterium Molecular Microbiology, pp. 29-69. Edited by T. Parish. Wydmondham, Norfolk. UK: Horizon Biosciences.

Saeed, A. I., Sharov, V., White, J., Li, J., Liang, W., Bhagabati, N., Braisted, J., Klapa, M. Currier T. \& other authors (2003). TM4: a free, open-source system for microarray data management and analysis. Biotechniques 34, 374-378.

Samten, B., Ghosh, P., Yi, A. K., Weis, S. E., Lakey, D. L., Gonsly, R., Pendurthi, U., Wizel, B., Zhang, Y. \& other authors (2002). Reduced expression of nuclear cyclic adenosine $5^{\prime}$-monophosphate response element-binding proteins and IFN-gamma promoter function in disease due to an intracellular pathogen. J Immunol 168, 3520-3526.

Sassetti, C. M., Boyd, D. H. \& Rubin, E. J. (2003). Genes required for mycobacterial growth defined by high density mutagenesis. $\mathrm{Mol}$ Microbiol 48, 77-84.

Schnappinger, D., Ehrt, S., Voskuil, M. I., Liu, Y., Mangan, J. A., Monahan, I. M., Dolganov, G., Efron, B., Butcher, P. D. \& other authors (2003). Transcriptional adaptation of Mycobacterium tuberculosis within macrophages: insights into the phagosomal environment. J Exp Med 198, 693-704.

Shalel-Levanon, S., San, K. Y. \& Bennett, G. N. (2005). Effect of oxygen, and ArcA and FNR regulators on the expression of genes related to the electron transfer chain and the TCA cycle in Escherichia coli. Metab Eng 7, 364-374.

Sharma, K., Gupta, M., Pathak, M., Gupta, N., Koul, A., Sarangi, S., Baweja, R. \& Singh, Y. (2006). Transcriptional control of the mycobacterial embCAB operon by $\mathrm{PknH}$ through a regulatory protein, EmbR, in vivo. J Bacteriol 188, 2936-2944.

Sherman, D. R., Voskuil, M., Schnappinger, D., Liao, R., Harrell, M. I. \& Schoolnik, G. K. (2001). Regulation of the Mycobacterium tuberculosis hypoxic response gene encoding $\alpha$-crystallin. Proc Natl Acad Sci U S A 98, 7534-7539.

Shi, L., Sohaskey, C. D., Kana, B. D., Dawes, S., North, R. J., Mizrahi, V. \& Gennaro, M. L. (2005). Changes in energy metabolism of Mycobacterium tuberculosis in mouse lung and under in vitro conditions affecting aerobic respiration. Proc Natl Acad Sci U S A 102, 15629-15634.

Sohaskey, C. D. \& Wayne, L. (2003). Role of narK2X and narGHJI in hypoxic upregulation of nitrate reduction by Mycobacterium tuberculosis. J Bacteriol 185, 7247-7256.

Stover, C. K., de la Cruz, V. F., Fuerst, T. R., Burlein, J. E., Benson, L. A., Bennett, L. T., Bansal, G. P., Young, J. F., Lee, M. H. \& other authors (1991). New use of BCG for recombinant vaccines. Nature 351, 456-460.

Talaat, A. M., Lyons, R., Howard, S. T. \& Johnston, S. A. (2004). The temporal expression profile of Mycobacterium tuberculosis infection in mice. Proc Natl Acad Sci U S A 101, 4602-4607.

Tam, C. \& Missiakas, D. (2005). Changes in lipopolysaccharide structure induce the $\sigma^{\mathrm{E}}$-dependent response of Escherichia coli. Mol Microbiol 55, 1403-1412.

Tufariello, J. M., Mi, K., Xu, J., Manabe, Y. C., Kesavan, A. K., Drumm, J., Tanaka, K., Jacobs, W. R., Jr \& Chan, J. (2006). Deletion of the Mycobacterium tuberculosis resuscitation-promoting factor Rv1009 gene results in delayed reactivation from chronic tuberculosis. Infect Immun 74, 2985-2995.

Tusher, V. G., Tibshirani, R. \& Chu, G. (2001). Significance analysis of microarrays applied to the ionizing radiation response. Proc Natl Acad Sci U S A 98, 5116-5121.

Voskuil, M. I., Schnappinger, D., Visconti, K. C., Harrell, M. I., Dolganov, G. M., Sherman, D. R. \& Schoolnik, G. K. (2003). Inhibition of respiration by nitric oxide induces a Mycobacterium tuberculosis dormancy program. J Exp Med 198, 705-713.

Walters, S. B., Dubnau, E., Kolesnikova, I., Laval, F., Daffe, M. \& Smith, I. (2006). The Mycobacterium tuberculosis PhoPR twocomponent system regulates genes essential for virulence and complex lipid biosynthesis. Mol Microbiol 60, 312-330.

Wu, Q. L., Kong, D., Lam, K. \& Husson, R. N. (1997). A mycobacterial extracytoplasmic function sigma factor involved in survival following stress. J Bacteriol 179, 2922-2929.

Wu, S., Howard, S. T., Lakey, D. L., Kipnis, A., Samten, B., Safi, H., Gruppo, V., Wizel, B., Shams, H. \& other authors (2004). The principal sigma factor sigA mediates enhanced growth of Mycobacterium tuberculosis strains in vivo. Mol Microbiol 51, 1551-1562.

Zahrt, T. C. \& Deretic, V. (2001). Mycobacterium tuberculosis signal transduction system required for persistent infections. Proc Natl Acad Sci U S A 98, 12706-12711.

Zahrt, T. C., Wozniak, C., Jones, D. \& Trevett, A. (2003). Functional analysis of the Mycobacterium tuberculosis MprAB two-component signal transduction system. Infect Immun 71, 6962-6970.

Zink, A. R., Grabner, W. \& Nerlich, A. G. (2005). Molecular identification of human tuberculosis in recent and historic bone tissue samples: the role of molecular techniques for the study of historic tuberculosis. Am J Phys Anthropol 126, 32-47.

Edited by: T. Parish 